\title{
¿SOCIOLOGÍA DE LA INFANCIA? Aproximaciones a un campo de estudio difuso
}

IVAN RODRIGUEZ PASCUAL

Universidad de Huelva

PALABRAS CLAVE ADICIONALES

Infancia, Familia, Socialización, Teoría sociológica

\section{ADDITIONAL KEYWORDS}

Chilhood, Family, Socialization, Sociological Theory.

RESUMEN. El estudio de la infancia y de su papel en el funcionamiento y reproducción de las sociedades humanas, incomprensiblemente, no ha ocupado un lugar central en la teoría sociológica. En este artículo se ofrecen algunas claves que permiten, sobra la base de las escasas aportaciones con las que contamos en relación al estudio sociológico de la situación social de los menores, tomar posiciones y reivindicar la infancia como un objeto de estudio netamente sociológico, bajo la hipótesis de que ésta no es sino una construcción social, cuya génesis debe ser estudiada en perspectiva histórica y social.

\section{INTRODUCCIÓN}

El estudio de la infancia como fenómeno social es un campo todavía insuficientemente explorado por parte de la sociología, aunque puede considerarse a la infancia como una construcción social, generada en el contexto de un proceso de cambio sociohistórico que ha modificado usos, concepciones y percepciones asociados a los menores. Como trataré de exponer en este artículo, la infancia así entendida, susceptible de un tratamiento sociológico, debería ser un campo preferente de investigación y teorización, sobre la base de una estrategia pluriparadigmática e interdisciplinar. A lo largo de las páginas siguientes analizaremos algunas de las aportaciones más relevantes que se han ofrecido para la interpre-

Revista Internacional de Sociología (RIS)

Tercera Época, n 26 , Mayo-Agosto, 2000, pp. 99-124. 
tación de este proceso de construcción del concepto de infancia, para después establecer una matriz de integración disciplinar y una primera clasificación de áreas de estudio relacionadas con ésta, sobre las que la sociología debería incrementar su atención.

\section{INFANCIA Y SOCIEDAD}

\section{¿Por qué preocuparse por la infancia?}

Aunque la pregunta con la que comenzamos esta reflexión pudiera parecer una cuestión meramente retórica, supone en realidad un punto elemental de partida para todos aquellos que, de una u otra forma, trabajamos en el ámbito de la infancia. De hecho, la motivación principal de escribir estas frases es, precisamente, responder a dos interrogantes básicos, a través de los cuales es posible formularse otros tantos; en primer lugar, surge la pregunta de si verdaderamente es posible el estudio de la infancia desde un punto de vista exclusivamente sociológico, es decir, ¿es posible una sociología de la infancia? En segundo lugar, es evidente que, aun en el caso de haber respondido afirmativamente a la primera de las cuestiones será necesario volver a interrogarnos sobre lo siguiente: en caso de que sea posible esa sociología, ¿cómo podemos delimitar con exactitud su campo de estudio? A su vez, será un paso previo ineludible para responder a estas cuestiones explicitar el proceso sociohistórico de construcción del concepto de la infancia y el estatus del menor, a través de la reconstrucción del mismo y de sus claves sociológicas.

Éstas pueden parecer cuestiones superficiales para todos aquellos que nunca hayan intentado un acercamiento a dicho objeto de estudio; sin embargo, desde el mismo momento en que ese acercamiento se produce — pongamos, por ejemplo, desde el punto de vista de un investigador de la realidad social - resulta palpable que la infancia es: a) un objeto difícil de definir, especialmente en cuanto su duración dentro del ciclo de vida de los individuos, b) un objeto difícil de investigar ${ }^{1}$, por cuanto se encuentra subsumido, a su vez, en otras realidades que también son estudiadas; así, por ejemplo, no son pocos los que piensan que el estudio de la infancia no debe ser sino una parcela dentro de lo que constituye el estudio de la familia como institución social, y por último c) un objeto comparti-

\footnotetext{
' Igualmente, la investigación de la infancia plantea en ocasiones problemas prácticos que derivan de la particularidad del objeto investigado, y que refieren a la aplicación de herramientas concretas de la investigación, como por ejemplo un simple cuestionario, que debe ser adaptado, por seguir con el ejemplo, al lenguaje de los menores.
} 
do, como lo son pocos, por múltiples disciplinas científicas no siempre permeables entre sí, y hablamos de la biología, la medicina, la psicología, la pedagogía, la sociología, etc.

Luego por tanto, el acercamiento a esa supuesta sociología de la infancia debe realizarse con cuidado. Ésa es la razón, por ejemplo, de que el título de esta reflexión aparezca entre signos de interrogación. En las páginas siguientes pretendo dar respuesta a las cuestiones anteriores, justificando la necesidad de prestar atención a la infancia como construcción social y aportando, en la medida que me sea posible, elementos que permitan delimitar el posible campo de estudio de una sociología de la infancia.

\section{Infancia y Sociedad a través de la historia: la génesis del estatus del menor}

El propósito de este apartado consiste en obtener una visión rápida del conjunto de aspectos que incluye la moderna concepción de la infancia, generalmente asociada a un ámbito de sobreprotección y control ${ }^{2}$. Es necesario emprender esta tarea antes de operacionalizar cualquier intento de definir el campo de estudio de la sociología de la infancia, en la medida que ésta es una construcción social y como tal es fruto de la acción de fuerzas combinadas a lo largo del tiempo. Lógicamente, el primer paso consiste en reconstruir los pasos del proceso a través del cuál ha sido posible la construcción de este ámbito de sobreprotección. Por otra parte, la simple afirmación de la existencia de un proceso tal, implica un ataque inevitable a la idea de la naturaleza del niño, tan extendida, por otra parte, en el discurso pedagógico, demostrando una vez más que para la sociología la idea de la naturaleza es un convidado de piedra (Lerena, 1985), un fantasma que esconde múltiples referencias a otros muchos fenómenos sociales entrecruzados. La cuestión es sencilla y ya ha sido formulada antes: la infancia no es un fenómeno "natural", directamente derivado del desarrollo físico, sino una construcción social que hace referencia a un estatus delimitado, incorporado dentro de una estructura social y manifestado en ciertas formas típicas de conducta, todas ellas relacionadas con un conjunto concreto de elementos culturales (Jenks, 1982).

El concepto de infancia con que frecuentemente operamos dista mucho del testimonio recogido por algunos de los autores que han trabajado este tema, y me refiero especialmente a las aportaciones que desde la historia se han realizado.

\footnotetext{
${ }^{2}$ En torno a la «sobreprotección» vinculada a la infancia resulta especialmente interesante consultar el trabajo de W. Corsaro (1997). Por otra parte, para rastrear el origen de la vinculación de la infancia a la idea de la "inocencia" y la necesidad de protección y control en el discurso pedagógico véase: (Varela, 1986).
} 
Especialmente traumática resulta la comparación, por ejemplo, cuando consultamos la Historia de la Infancia que Lloyd De Mause nos propone, donde incluye los principios de su ya conocida teoría psicogénica. La infancia que De Mause (1982) describe es la infancia de las fajaciones, los malos tratos, los abusos sexuales, el dolor aplicado a los niños, los abandonos, el infanticidio, etc. Es preciso reconocer que, en mi opinión, lo valioso de la aportación de De Mause acaba donde comienzan las limitaciones de su método psicoanalítico, que prescinde en demasiadas ocasiones de factores claves como la esfera cultural, el contexto demográfico, o los referentes sociohistóricos concretos, al tiempo que tiende a juzgar fenómenos que sólo pueden ser interpretados a la luz de una determinada formación social y cultural como sucesos "puestos al día", con la distorsión que esto conlleva. Por otra parte, aunque su trabajo es fundamental, en cuanto se propone el descubrir las verdaderas condiciones de existencia de la infancia a lo largo de la historia, no profundiza en las claves que explican los mismos fenómenos que denuncia.

La cuestión queda planteada pero no resuelta, precisamente por obviar un detalle que parece evidente desde una perspectiva sociológica: la ausencia de un equivalente exacto en dicho contexto histórico de nuestra moderna concepción de lo que es la infancia y las obligaciones y tareas que comporta. Como otros autores han señalado hace ya tiempo, es precisamente el descubrimiento de ese nuevo sentimiento de la infancia (Ariès, 1987) ${ }^{3}$, lo verdaderamente nuevo en el proceso histórico que lleva desde el infanticidio tolerado a la firma de la primera Declaración de Derechos del Niño en 1959. El propio De Mause admite la existencia de un proceso al que adjudica distintas fases ${ }^{4}$. En general, y sin temor a adelantarnos en los acontecimientos, Siguiendo al profesor Morente, podemos nombrar tres grandes momentos en ese proceso que constituyen puntos de inflexión en la evolución de la infancia (Morente, 1997: 40-41): a) el cambio demográfico hacia una sociedad más vieja, b) la integración del menor en un espa-

\footnotetext{
${ }^{3}$ Precisamente, el propio Lloyd De Mause cita a Ariès como sustentador de una tesis opuesta a su particular interpretación de los hechos históricos, cita en la que puede leerse entre líneas un cierto reproche a la actitud mucho más "templada" del segundo con respecto al problema del infanticidio y otras prácticas lesivas para la infancia.

${ }^{4}$ Que el resume de la siguiente forma: 1) Infanticidio (Antigüedad-siglo IV); 2) Abandono (siglos IV-XIII); 3) Ambivalencia (siglos XIV-XVII); 4) Intrusión (siglo XVIII); 5) Socialización (siglos XIX-XX); 6) Ayuda (mediados siglo XX). Según este esquema, a la última etapa le corresponde el punto de partida de una actitud verdaderamente responsable para con las necesidades de la "naturaleza" del niño. De acuerdo con sus propias palabras, que más parecen las de un pedagogo que las de un historiador, su resultado es un niño amable, sincero, que nunca está deprimido, que nunca tiene un comportamiento initativo o gregario, de voluntad firme y en absoluto intimidado por la autoridad (De Mause, 1982: 89-90).
} 
cio de formación (escuela), y c) la interrupción del modelo sencillo de reciprocidad entre generaciones (asistencialismo), sustituido por la aparición de la seguridad social.

En este sentido, la aportación de Ariès está más cercana al propio núcleo del pensamiento sociológico. El autor parte de la tesis de una indiferenciación del mundo infantil y adulto durante el antiguo régimen, con un amplio abanico de posibilidades de sociabilidad fuera del hogar ${ }^{5}$, que la propia revolución de la modernidad y la sociedad industrial se encarga de confinar en un nuevo espacio, el de la familia. Por supuesto, también el autor cataloga y describe múltiples prácticas que, a nuestros ojos, no parecen sino aberraciones y, según los casos, auténticas torturas. Lo interesante, sin embargo, es la reconstrucción e interpretación histórica del proceso, que permite seguir los pasos a ese moderno sentimiento de la infancia, condición por otra parte indispensable si verdaderamente queremos poner en práctica una sociología de la infancia.

La tesis de Ariès, no obstante, también ha sido criticada, aludiendo en ocasiones al hecho de una supuesta ignorancia de la primera infancia y la exageración en cuanto a la escasez de control socializador del núcleo familiar (Segalen, 1992; Hufton, 1992). En cualquier caso, contiene en sí misma el valor de haber apreciado lo que en muchos diccionarios de pedagogía y ciencias de la educación no es sino una "realidad invisible", es decir, la construcción social del concepto moderno de infancia. Especialmente relevante me parece la cronología del autor, que hace coincidir la aparición de un cambio apreciable con respecto a la infancia con la todavía incipiente sociedad burguesa e industrial, en los siglos XVII y XVIII, preparando un giro que iba a tener en el XIX, con los primeros intentos serios de escolarización masiva, su punto álgido. Las palabras del autor son de una claridad meridiana a este respecto (Ariès, 1987: 56):

Parece como si a cada época le correspondiese una edad privilegiada y una periodicidad particular de la vida humana: la "juventud" es la edad privilegiada del siglo XVII; la infancia del siglo XIX, y la adolescencia del siglo XX.

Efectivamente, las revoluciones burguesa e industrial traerán consigo todas las transformaciones que hoy bien conocemos, y muchas de ellas guardarán una especial relación con la reorganización de la estructura de la institución familiar, y por ende, de la infancia. Es en este sentido que afirmo que, verdaderamente, no se concibe un estudio del cambio histórico y social en la infancia sin estudiar, paralelamente, los cambios en las formas de vida familiar, que sufren un profun-

\footnotetext{
5 "Las relaciones afectivas y las comunicaciones sociales se consolidaban pues fuera de la familia, en un círculo denso y muy afectuoso, integrado por vecinos, amigos, amos y criados, niños y ancianos, mujeres y hombres, en donde el afecto no era fruto de la obligación, y en el que se diluían las familias conyugales" (Ariès, 1987: 11).
} 
do reajuste del que aún nos sorprendemos hoy día. Parece ya superada, no obstante, la vieja concepción que pretendía una transición lineal transparente desde una familia extensa a otra nuclear, más evolucionada y acorde con la realidad de la sociedad industrial. La coexistencia entre ambos modelos, y otros muchos intermedios, y el paso de buena parte de las familias por ambos polos durante su ciclo de vida parece haber sido, en realidad, la tónica dominante durante buena parte de la edad media y el antiguo régimen (Burguiére, 1998; Anderson, 1982), de la misma forma que no es menos cierto que la idea de que la familia constituye el ámbito por antonomasia de la procreación y la tutela de la infancia es relativamente reciente, existiendo en la Edad Media y el Antiguo Régimen frecuentes ejemplos que se salen fuera de esta norma (Morente, 1996).

No obstante, parece claro que lo que antes era un espacio lleno por la actividad de la vida pública, en el que el niño podía desenvolverse con una libertad que hoy provocaría encendidos debates morales, poco a poco fue contrayéndose hasta cuajar en la idea del hogar, a salvo de miradas del exterior, recluido en sí mismo y, esto es lo importante desde el punto de vista de la infancia, el lugar por excelencia de control y socialización del niño, que parece haber quedado atrapado, curiosamente junto con su madre (otra de las grandes figuras "mudas" de la historia) en él. En este sentido, es indudable que la aportación de Norbert Elias y sus estudios sobre el proceso de la civilización son esclarecedores, por cuanto desvela el papel que la coacción y el aprendizaje del pudor y la autorepresión han jugado en la formación de la infancia moderna. Partiendo del texto de Erasmo (precisamente, una guía para la educación infantil) Civilitate Morum Puerilium, el autor articula una explicación de cómo la "civilización" constituye un principio de transformación del comportamiento que opera de generación en generación, a través de un mecanismo psicogenético y sociogenético (Elias, 1989).

El valor del estudio de Elias, en lo que respecta a la infancia y su evolución histórica, no es otro que el de comprender los mecanismos psico y sociogenéticos que operan en la transmisión cultural en el proceso de generaciones, en este caso, como señala el autor, en la dirección de un creciente autocontrol que eliminan los amplios márgenes de conducta medievales y que puede ser la base de la reducción de ese espacio de sociabilidad del que, como ya hemos visto, nos habla Ariès. La aparición del pudor ante nuestros actos en la esfera pública, la imposición de rígidas normas de socialización, la actitud de prudencia hacia el extraño..., son todas secuencias de un mismo movimiento que culmina en la aparición de lo que se ha dado en llamar la sociedad íntima ${ }^{6}$, que guarda no poca relación con el nuevo sentir hacia la infancia y su caracterización como una etapa de necesaria sobreprotección. En realidad, las tesis de Elias sobre la socialización en la autocoacción retoma la vieja idea freudiana en la que filogénesis y sociogénesis se encuentran encadenadas por el lazo de la represión de las pulsiones inmediatas en el ser humano (Freud, 1970), tarea que, como Freud señala con frecuencia, tiene una especial significación durante los primeros años de vida. 
En cualquier caso, venimos hablando de un "proceso", en el cual es posible detectar un nuevo interés y una nueva actitud hacia aquello que hoy llamamos infancia, pero esto no debe ocultar el hecho de que no ha sido un proceso unitario y homogéneo, subsistiendo importantes diferencias al analizar la situación de la infancia en diferentes estratos sociales. Durante mucho tiempo se ha sostenido que dentro de las clases populares, y paralelamente a la aparición de ese nuevo sentimiento del que ya hemos hablado, era posible encontrar restos de una antigua sociabilidad que no concuerda con algunos de los aspectos comentados hasta ahora. El hecho de que los chicos de clase obrera, por poner un caso, siguiesen socializándose más como aprendices en un taller que como niños vulnerables, o fueran objeto en mayor medida de la curiosa práctica (de especial relevancia en Inglaterra) del lyfe-cycle servant, da buena cuenta de ello (Burguiére, 1998: 48). Esta distinción encuentra un momento agudo de expresión ya en el XIX, cuando la sociedad industrial ve enconizado el problema de la pobreza urbana y las diferencias entre clases. Así, no debemos olvidar que el modelo de socialización basado en el hogar, la autoridad paterna, y la sobreprotección del niño difícilmente puede ser aplicado en el caso de las familias obreras, donde, en muchos casos, los menores son aún apreciados, básicamente, por constituir una importante mano de obra y nuevos ingresos familiares. Por ello, será necesario prestar una especial atención a un momento clave en la aparición de la niñez tal y como la entendemos en la actualidad, y que viene a coincidir, como ya he adelantado en párrafos anteriores, con el inicio de la escolarización obligatoria y el surgimiento de los primeros sistemas de atención a la infancia.

Efectivamente, el surgimiento de los sistemas educativos (al menos en su vertiente compulsiva en cuanto a la escolarización del alumnado) marca un punto de inflexión en la situación social de la infancia, que coincide con un giro copernicano en la forma de interpretar y actuar sobre esta etapa del ciclo de vida de los individuos. La tendencia observada durante el siglo XVII y parte del XVIII es a mostrar un escaso interés por parte de los Estados hacia la infancia, siendo muy escasa la legislación producida en este sentido (Lebrun, 1998), situación que cambiará en la segunda mitad del XVIII con la introducción de nuevas ideas ilustradas, la publicación de diversos textos sobre la infancia - de entre los cua-

'La sociedad íntima es la sociedad en la que la esfera privada cobra protagonismo, y <<ésta no es más que el lugar donde "uno" toma conciencia de su existencia frente a "los muchos", el nido donde el individuo tiende a desarrollar sus potencialidades, lejos del ruido de la colectividad $>$ (Béjar, 1990: 15). Por otra parte, dado que el tema de la conexión entre la sociedad íntima y el moderno concepto de infancia excede las pretensiones de este ensayo, pero resulta sumamente interesante es recomendable consultar, aparte de la obra de Elias ya reseñada en estas páginas, otros dos volúmenes: el libro de Richard Sennett (1978) y el de Robert N. Bellah et.al. (1989). 
les sea, probablemente el Emilio de Rousseau el más conocido-, y la aparición, en fin, de un nuevo sentir que se traduce en una clara tendencia hacia el control de la vida de los niños, bajo el argumento de la necesidad de acabar con prácticas como el infanticidio, el abandono, etc. Desde este punto de vista, la escolarización constituye una herramienta privilegiada que se circunscribe en el ámbito del control y la normalización, pasos esenciales para regularizar lo que antes escapaba al control estatal. Esta tesis ha sido comúnmente defendida desde la Sociología de la educación ${ }^{7}$ y contiene además un matiz referente a las diferencias de clases en la todavía incipiente sociedad industrial, especialmente presente en la escuela marxista, en el que no vamos a entrar. Baste señalar, como ya ha hecho Ariès en la parte de El niño y la vida familiar en el antiguo régimen dedicada a la educación, que la entrada en juego de la enseñanza reglada viene a acabar con la fragmentación y la multiplicidad de las escuelas medievales ${ }^{8}$, para imponer un sistema que se sustenta en un concepto seminal: la disciplina (Ariès, 1987).

Precisamente, la aparición de la escuela y de los primeros movimientos hacia un orden asistencial hacia los menores constituye un momento crítico en esa construcción social del sentido de la infancia de la que hablamos. Por otra parte, el nacimiento de la sociedad industrial supone la cristalización de un mundo de desigualdades sociales que va a afectar radicalmente al niño y a la familia. Así, hablemos en primer lugar del nacimiento de una necesidad de control con respecto a los menores, revelando un interés por aspectos educativos poco conocidos en el Antiguo Régimen.

Normalmente, es usual encontrar múltiples referencias a este movimiento de creación de instituciones que, como la escuela o el hospicio, van a incorporar al niño en sus estructuras de socialización. Aunque es exagerado afirmar que la familia pierda, como consecuencia de este proceso, su función como agente de socialización, sí resulta interesante resaltar en qué medida la antigua sociabilidad extrafamiliar es sustituida no por una total reclusión en al ámbito doméstico sino por una marcada presencia institucional. Presencia que, en la mayor parte de los casos, es analizada por parte de la sociología aplicando los conocidos principios de la microfísica del poder (Foucault, 1975), especialmente en el caso de las instituciones asistenciales. Resulta innegable que, en muchos casos, ese espíritu de "asistencia" a los menores, parece más un espíritu de "resistencia"

\footnotetext{
${ }^{7} \mathrm{~A}$ modo de ejemplo, baste la lectura del texto de Julia Varela "Elementos para una genealogía de la escuela primaria en España", en Ortega (1989), donde al análisis de la aparición de la enseñanza primaria y la propia figura del maestro gravita en todo momento alrededor de la noción de control.

${ }^{8}$ Para una caracterización histórica de la construcción de un tiempo escolar normalizado véase Viñao (1998).
} 
ante las consecuencias que podían derivar de una inadecuada socialización de los mismos. Por ello, se señala con frecuencia el hecho de que bajo el humanismo pretendidamente reformista que mueve este tipo de instituciones encontramos una clara necesidad de vigilar y poner en vereda un colectivo potencialmente peligroso, muy alejado de los niños que presenta el modelo familiar típicamente burgués, asentado sobre un predominio afectivo y el espacio cerrado del hogar. El texto siguiente, de principios de este siglo, ejemplifica bien esto a lo que nos referimos":

Si dejamos abandonados, sin cuidarnos de ellos, a esos miles en peligro moral y delincuentes, si no dedicamos a la obra de su redención nuestra actividad y nuestro dinero, en un porvenir más o menos lejano, cuando su potencia criminal haya alcanzado un desarrollo adecuado pondrán en peligro nuestra vida y nuestros bienes, o se apoderarán de ellos por la fuerza o por la astucia [... y como también habrán otros menores que, sin ser propiamente criminales, caerán en la vagancia y en la miseria, necesitarán ser acogidos en asilos y hospitales, gastos que también tendremos que pagar.

De acuerdo con este enfoque predominantemente utilitario, la infancia peligrosa es la infancia del abandono, la niñez que se define en términos de vagabundeo y exposición a perniciosos influjos que terminan en la conducta delictiva. Nótese que no es éste un ejemplo aislado. Muy al contrario, es posible encontrar múltiples referencias a esta visión utilitaria dentro de la sociología de la educación, a las que aquí no vamos a hacer referencia por no constituir el tema central de nuestra reflexión ${ }^{10}$. En cualquier caso, este tipo de prácticas encuentran una explicación al contrastar los distintos modelos de socialización que prevalecen para la infancia en el contexto de la época. Así, hemos señalado antes el hecho

"Cuello Calón (1917), citado en Davila, Pauli et al., (1991: 236).

${ }^{10}$ Por encontrar se encuentran incluso huellas de este tipo de prácticas incluso entre los clásicos de la literatura posterior a la revolución industrial. El siguiente pasaje, por ejemplo, ha sido extraído de la obra Oliver Twist, de Charles Dickens: "y no crean los enemigos del Sistema, que durante su prisión se privase a Oliverio de las ventajas del ejercicio, del placer de la sociedad, o de los consuelos de la religión. En cuanto a lo primero, como el tiempo era hermoso y frío, se le daba permiso para lavarse todas las mañanas con el agua de la fuente que había en un patio, en presencia del señor Bumble, quien para impedir que se constipara activaba en el chico la circulación de la sangre por medio de frecuentes bastonazos. Por lo que hace a la sociedad, llevábanle todos los días al refectorio de los niños, donde se le administraba un duro correctivo para el buen ejemplo y edificación de los demás; y últimamente, como consuelo religioso, hacíanle entrar a patadas todas las noches en la sala, llegada la hora de rezar, y se le permitía oír la oración con sus compañeros, corregida y aumentada por el consejo, en el cuál recomendábase la virtud, la docilidad y la obediencia para preservarse de las faltas y vicios de Oliverio Twist, que era un hijo de Satanás patrocinado por el diablo". Editorial Planeta, 1988, pg. 24. 
de que las diferencias en la socialización de las clases populares y las acomodadas es grande, y en términos de desigualdad a veces casi abismales. Desde un punto de vista burgués, en el que la infancia, en virtud de modernas teorías pedagógicas y la propia práctica familiar, debe ser retirada del ámbito de lo público y protegida al calor del hogar y la disciplina paterna, la vida de los chicos y chicas de otros estratos sociales, especialmente los de la clase obrera, debe interpretarse ineluctablemente en términos de peligrosidad social. Finalmente, será éste paradigma socializador el que se imponga en Occidente, impregnando de aquí en adelante el concepto de infancia que nos a va a legar la modernidad; la infancia de la protección, del afán normalizador en los comportamientos del niño, de la reclusión íntima y doméstica, en definitiva.

A su modo, también las escuelas suponen un cambio radical en la socialización secundaria de la infancia. No sólo por el hecho de que sustrae al niño de otros posibles agentes socializadores, como ocurriría en el caso de que éste permaneciese en el seno familiar o se integrase dentro de la práctica del lyfe-cicleservant u otra similar, sino también por imponer, dentro del período de vida escolar del niño, una medida de los tiempos y los movimientos, un orden escolar, una forma, en definitiva, de aprendizaje normalizado y sistematizado" que se ve sancionado por un conjunto de normas y de fines comúnmente aceptados (una cultura escolar, en suma) y una sistematización instrumental de los contenidos enseñados (curriculum) $)^{12}$. El tiempo que el niño pasa en la escuela se caracteriza, como bien señala Phillip Jackson, por su consistencia (Jackson, 1991), concepto referido en última instancia al orden y la disposición de los quehaceres cotidianos en las aulas, que resulta de la necesidad de proporcionar una «socialización institucional $»^{13}$, diferente de ese primer contacto socializador que el niño recibe en el grupo familiar. De alguna forma, aunque escuelas y hospicios albergan dinámicas de funcionamiento distintas - mucho más cercanos los segundos a lo que se conoce en sociología como instituciones totales (Goffman, 1972)— denotan un mismo fenómeno social: el desarrollo de un creciente interés por parte del Estado en lo referente a asuntos que afectan a los menores, trascendiendo la ya tradicional, como hemos visto en páginas precedentes, cuasi-indiferencia que lo había caracterizado hasta el momento. Más allá de la simple preocupación

\footnotetext{
"Que Anthony Giddens utiliza como ejemplo de regionalización en su obra: La constitución de la sociedad (1995).

${ }^{12}$ Quizás sea por ello por lo que Antonio Viñao (1998: 8) escribe, a propósito de la modulación del tiempo escolar a lo largo del siglo XIX, que este reviste una triple naturaleza: "a) como medio disciplinario, b) mecanismo de organización y racionalidad curricular y c) instrumento de control externo".

${ }_{13}^{13}$ Tal y como la define Julio Iglesias de Ussel (1985).
} 
por el infanticidio, o el "mimoseo" de Ariès, encontramos un interés por comprender y desarrollar nuevos conocimientos sobre el niño, que ya no es un adulto en potencia, sino un ser social en potencia, y como tal, deben extremarse los cuidados en su socialización.

En las páginas siguientes veremos como podemos emplear todas las consideraciones previas sobre el proceso de construcción social del concepto de infancia, a la hora de reivindicar éste como un legítimo objeto de estudio sociológico, al tiempo que trataremos de desvelar las implicaciones que derivan de este objetivo.

\section{LA INFANCIA: CONSTRUCCIÓN DE UN CAMPO DE ESTUDIO}

\section{Algunas cuestiones relevantes. ¿Una o muchas infancias?}

Acentuar, como hemos hecho hasta ahora, el aspecto de construcción sociohistórica que la infancia presenta a los ojos del sociólogo nos ha servido para reclamar un punto de vista distinto, que contemple fenómenos sociales complejos que vienen a cristalizar en un determinado sentir o percibir la niñez en nuestra sociedad. Sin embargo, nuestro intento no debe ni puede ser confundido con un afán por forzar los hechos históricos y sociales de tal forma que parezca que la infancia actual es la culminación de un proceso natural y necesario, el punto y final de una evolución que deriva de la misma naturaleza superior de aquello que se presenta más complejo, más "evolucionado". Ha sido ése un error que ya otros han cometido antes en muchos otros campos, y que aquí intentaremos no repetir en la medida de lo posible. Buscamos, por otra parte, salir al paso de otro error comúnmente denunciado como tarea habitual del quehacer sociológico, el reduccionismo, que como Miguel Beltrán señala, "escamotea lo específico de la realidad social en favor de otras realidades diferentes, sobre todo de la realidad individual y de la realidad biológica" (Beltrán, 1991: 81). Por esta razón nos entretenemos en señalar qué es lo que hace a la infancia por un lado un fenómeno complejo, no siempre bien subsumido bajo campos genéricos como el de "crecimiento" o "educación", y por otro un fenómeno que reclama una interpretación sociológica en mayor medida de lo que ha venido ocurriendo hasta ahora.

Llegados a este punto, se hace necesario señalar el hecho de que el contemplar la infancia desde un punto de vista sincrónico (habiendo dejado ya atrás las breves nociones de historia de la infancia que hemos reseñado en epígrafes anteriores) no deja de revelarnos su marcado carácter de diversidad. Dicho de otra forma, aunque parezca paradójico, no es posible hablar de una infancia sino en un sentido muy amplio y genérico - como ocurre con tantos conceptos sociológicos- porque sería más justo hacer referencia a las múltiples manifestaciones del fenómeno. No estamos hablando ya de un proceso sociohistórico, sino de una situación social aprehendida, si se quiere, de forma muy intuitiva. Es eviden- 
te que la infancia de un menor en un suburbio de Los Angeles y la de un miembro de la realeza británica son ciertamente disímiles. Y aunque el ejemplo es excesivo podemos encontrar otros muchos aplicables a nuestra sociedad. Así, desde lo más pequeño (el ambiente familiar) a lo más amplio (la clase social, el género, o los sistemas sociales en su conjunto, por ejemplo) todo influye en hacer que el fenómeno de la niñez contemplado desde una perspectiva sociológica adopte distintas formas que deben, igualmente, ser tomadas en consideración.

Tomemos, como ilustración, un primer nivel; hablemos, por tanto, de un aspecto puramente demográfico: el peso del grupo de edad de 0 a 14 años en el conjunto de la población. El siguiente cuadro recoge de forma resumida la información de la UNESCO en cuanto al crecimiento demográfico de dicho grupo en distintos lugares del planeta para el período 1970-1999 (tabla 1).

Las diferencias son claras. Los países que la UNESCO agrupa bajo la categoría "desarrollados" marcan una intensa tendencia decreciente en la presencia de sujetos entre 0 y 14 años, como corresponde a una dinámica demográfica caracterizada generalmente por una natalidad habitualmente por debajo del índice de reproducción de generaciones. Así, se produce una paradoja, por cuanto es en estas sociedades desarrolladas, en las que los niños cada vez representan un grupo más minoritario dentro de la población total, en las que se ha producido un auge notable del interés de pedagogos, educadores, psicólogos, etc. por los aspectos relacionados con la infancia. No ocurre lo mismo, sin embargo, si desplazamos nuestro punto de vista hasta el continente africano, donde la tendencia, si bien común, presenta un contraste tan grande con la anteriormente citada en sus cifras - aunque en el caso de los países del Norte de África ya lejos de la denominada "fecundidad natural" (Arango, 1993) - lo que muchas veces es percibido como una "amenaza demográfica" por parte de dichas sociedades desarrolladas. En general, la tendencia a la reducción del peso demográfico de los menores

Tabla 1.

Población de 0 a 14 años (1970-1999).

\begin{tabular}{lcccc}
\hline \multirow{2}{*}{$\begin{array}{l}\text { Continentes, grandes } \\
\text { regiones y grupos de países }\end{array}$} & \multicolumn{4}{c}{ Población de 0 a 14 años (\% sobre el total) } \\
\cline { 2 - 4 } & 1970 & 1980 & 1990 & 1999 \\
\hline África & 44,8 & 44,9 & 45,8 & 38,4 \\
América & 36,3 & 32,3 & 30,4 & 32,2 \\
Asia & 40,3 & 37,6 & 32,8 & 34,4 \\
Europa (inc. URSS) & 26,3 & 23,2 & 21,7 & 18,7 \\
Países Desarrollados & 26,9 & 23,5 & 21,8 & 22,7 \\
\hline
\end{tabular}

Fuente: Elaboración propia a partir de: Unesco, Statistical Yearbook , años 1990 y 1999. 
parece tener lugar, con mayor o menor intensidad, en todos los grupos de la tabla, augurando una extensión planetaria del fenómeno de la conversión de la infancia en un grupo minoritario de población.

Más allá de las diferencias puntuales que el cuadro señala, es interesante destacar cómo la infancia, incluso en la más básica manifestación del nacimiento, se encuentra incardinada en un conjunto de circunstancias y tendencias que son comunes a una sociedad o conjunto de sociedades, de tal forma que no es posible separarla siquiera del contexto social en el que se produce el fenómeno. De esta forma, la infancia no es simplemente un estado previo a la sociedad, un momento de socialización que conduce a la posterior vida societal o comunitaria, sino que desde un primer instante constituye una cristalización concreta de estructuras, procesos e instituciones sociales que van a dar lugar, efectivamente, a la materialización de un ser social. En este sentido, una visión que tiende a resaltar esos mismos aspectos sociales que aquí venimos señalando es la de Emile Durkheim, que aunque no se refiere concretamente a la infancia, al hablar de la educación como proceso escribe (Durkheim, 1990: 53) "la educación es la acción ejercida por las generaciones adultas sobre aquellas que no han alcanzado todavía el grado de madurez necesario para la vida social. Tiene por objeto el suscitar y desarrollar en el niño un cierto número de estados físicos, intelectuales y morales que exigen de él tanto la sociedad política en su conjunto como el medio ambiente específico al que está especialmente destinado", una visión sin duda sustentada sobre una concepción puramente sociológica de la vida humana, así como de su proceso de crecimiento y maduración, proceso que pasa, necesariamente, por la figura del niño por cuanto éste encarna toda la potencialidad que ese Yo social puede proporcionar. En el fondo, la situación paradójica de ese doble movimiento proviene del propio carácter homogéneo/heterogéneo que el mismo proceso de socialización posee, y constituye, no cabe duda, la fuente de la riqueza en términos de complejidad del fenómeno, que encontramos cada vez que tratamos de acercarnos a la infancia como objeto de estudio por parte del sociólogo.

Por otra parte, el peligro de considerar al niño como un producto social en sentido estricto nos lleva directamente a un error en el que, a decir de algunos, la sociología ha venido incurriendo tradicionalmente; esto es, considerar a la infancia desde el punto de vista de la aceptación pasiva de la socialización paterna (Huber y Spitze, 1988), aunque por extensión podríamos decir de la socialización en general. Diríase, por tanto, que el movimiento infancia-sociedad es doble, por cuanto nunca deja de hundir sus raíces en un conjunto de fuerzas que son, al mismo tiempo, constrictoras y liberadoras, determinantes e indeterminadas. Dejemos expresarse a aquellos que abogan por este carácter no determinista de la infancia (Erikson, 1993: 364-365):

Toda sociedad está formada por hombres que se encuentran el proceso de dejar de ser niños para convertirse en progenitores. Para asegurar la continuidad 
de la tradición, la sociedad debe prepararse desde temprano para esa tarea en sus niños; y debe encargarse de los residuos inevitables de infantilismo en sus adultos. Se trata de una vasta tarea, sobre todo considerando que una sociedad necesita de muchos seres que puedan seguir, unos pocos que puedan conducir, y algunos capaces de hacer ambas cosas, en forma alternada o en distintas áreas de la vida [... cada generación debe desarrollarse a partir de su propia infancia y, superando su tipo particular de infancia, debe desarrollar otra nueva, potencialmente promisoria y potencialmente peligrosa.

\section{¿Sociología de la Infancia u otras Sociologías?}

Tal y como hemos venido estudiando a lo largo y ancho de esta reflexión, la infancia constituye el fruto de un devenir sociohistórico y se inserta, ineluctablemente, en un contexto social. A un nivel más concreto, es evidente que la infancia constituye un momento del ciclo de vida del ser humano, íntimamente conectado con la institución social de la familia, por cuanto es en el marco generalmente reducido de ésta en el que crecemos y nos desenvolvemos, en nuestra calidad de "aprendices" de la vida social. Sobre la familia, desde luego, se ha escrito mucho, y nadie se sorprende de que exista una "Sociología de la Familia" como campo de estudio consolidado dentro de la Sociología. Además, en estas mismas páginas, hemos estudiado cambios concretos operados en el concepto de infancia que no se entendían sin analizar paralelamente los cambios producidos en la estructura familiar. En un principio, por tanto, no parecería descabellado afirmar que el estudio de la unidad familiar incluye el estudio de la niñez, y que ésta no necesita de una especial atención sociológica, o al menos no más que cualquier otro objeto de estudio igualmente susceptible de ser abordado sociológicamente.

La realidad, sin embargo, es otra desde el momento en que el estudio de la infancia, de la socialización intra y extrafamiliar, de la conexión entre estructura social y crecimiento, educación, etc., lejos de haber sido integradas en la reflexión sociológica sobre la familia, se han difuminado, unas veces "suplantadas" por visiones simplistas de la niñez, otras abandonadas a consideraciones lejanas a nuestra disciplina (como las efectuadas por pedagogos o psicólogos), y otras, simple y llanamente, desestimadas como aspectos de interés desde el punto de vista científico, o al menos sociológico.

En cuanto al resto, resultaría difícil resumir aquí todos los problemas por los que la Sociología, y más concretamente en otros campos, como la Sociología de la Educación, ha atravesado en su peculiar lid con otras ciencias afines, de entre las cuales es obligado destacar la confrontación con el discurso pedagógico, campo en el que la Sociología española produjo algunas de sus más brillantes aportaciones en boca del ya desaparecido Carlos Lerena ${ }^{14}$. Baste señalar, a modo de hilo narrativo para futuras explotaciones de este tema, el obstáculo que supone el 
punto de vista de algunas disciplinas que reducen un fenómeno que para la investigación sociológica es complejo y en extremo ramificado (hablamos de la propia infancia) a una mera sucesión de momentos evolutivos (como viene ocurriendo con la psicología, y muy especialmente con la psicología evolutiva) o a ideas abstractas pretendidamente asépticas que, en realidad, revelan un conocimiento parcial de la esencia misma de los procesos sociales de desigualdad o poder, por poner un caso (la idea de "naturaleza" o "esencia" del niño, muy presente en la pedagogía). Probablemente haya sido en el estudio de la escolarización donde más crudamente se han hecho evidentes estas diferencias, por más que desde la sociología, y especialmente desde la rama más crítica, se ha señalado la enorme importancia que tiene el contemplar ésta "dentro de un marco de relaciones, de considerarla conectada - fundamentalmente- con las relaciones de dominación y explotación (y con las luchas en contra de ellas) de la sociedad en general" (Apple, 1996: 29), o lo que es lo mismo, poner el acento en la estructura social y aumentar el campo de visión de una vez por todas.

En cualquier caso, vista la situación, considero suficientemente justificado el tratar la infancia como un objeto de estudio específico con suficiente consistencia como para dedicarle nuestra atención, aunque ésta no tenga porqué estar engarzada forzosamente dentro de la Sociología de la Familia o de la Educación, ni se niegue, por razones evidentes, que guarde una estrecha relación con ellas.

\section{¿Cómo definir la Infancia?}

Lo que a primera vista pudiera parecer el momento más elemental de la investigación sociológica de la infancia es también el más complicado. Y es así porque, habitualmente, los intentos de definir el fenómeno chocan con la necesidad de acotar el mismo en términos cronológicos, labor que se torna cuando menos extremadamente complicada desde el mismo momento que el fenómeno a estudiar muestra manifestaciones muy diferentes en función del contexto cultural en el que se encuentre ${ }^{15}$. En nuestra sociedad occidental modernizada hemos partido de la base de aplicar un tramo de edad que, por lo general, coincide (al menos en su límite superior) con el período de escolarización obligatoria, por

\footnotetext{
${ }^{14} \mathrm{~A}$ este respecto nunca está de más recordar que algunas de las más ingeniosas críticas del citado a los movimientos de renovación pedagógica las podemos encontrar en (Lerena, 1986; 1985).

${ }^{15}$ Una posible solución pasa por olvidar la necesidad, no siempre justificada, de encerrar la realidad social en definiciones con pretensiones generalidad o universalidad y ser un poco más operativos, reconociendo que el límite cronológico puede variar y debe ser establecido sólo en el marco concreto de la investigación y atendiendo, de forma prioritaria, al objeto investigado.
} 
cuanto escuela e infancia parecen ser dos entidades que, cada vez en mayor medida, aparecen asociadas; así, alrededor de los 14 años solemos situar el final de esta etapa del ciclo de vida ${ }^{16}$.

Sin embargo, creo más útil comenzar por plantearnos incluso la pertinencia de la misma pregunta que da título a este apartado. No puedo estar menos de acuerdo con las afirmaciones de Giovanni Levi y Jean-Claude Schmitt (1996: 8) cuando se enfrentan a un problema parecido al tratar el problema genérico de la juventud: "Semejante edad de vida no puede hallar una delimitación clara ni en la cuantificación clara ni en una definición jurídica, hasta tal punto que estimamos de escaso provecho el dedicarnos, después de otros, a la búsqueda de unas fronteras harto señaladas ya". No se trata de negar que la infancia, como tal fenómeno social, carezca de un lugar en el tiempo o en el espacio o que éstos sean desconocidos, pero desde el mismo momento en que hemos insistido en la necesidad de admitir que depende en gran manera del contorno cultural y social en el que se encuentra, sería muestra de gran arrojo y de igual insensatez pretender encorsetar el problema dentro de unos límites cronológicos precisos y, al mismo tiempo, pretender que ésta fuera una acotación válida a modo de "universal cultural". De esta forma, se indicarán aquellas características que pueden ayudar a identificar la infancia, otorgando a esta identificación la seña de la flexibilidad como primer y más importante rasgo del mismo. Así mismo, vaya por delante que si no existiera un conjunto de características comunes no tendría sentido su estudio como objeto diferenciado dentro del estudio de la sociedad en general.

En principio, antes de acometer la tarea antes señalada, conviene concretar por qué en diferentes momentos de esta reflexión he manifestado que la infancia constituye un objeto de estudio "disperso" o "difuso", y en cierta medida "abandonado", dentro del campo de la sociología.

Disperso, porque como ya hemos visto el objeto ha sido fragmentado, parcelado, y repartido entre muy distintas disciplinas que abarcan desde las ciencias de la salud (el caso de la pediatría, por ejemplo) a la psicología, la pedagogía, o el trabajo social. La fragmentación interdisciplinar favorece el estudio del fenómeno desde el mismo momento en que la descomposición de un problema general en otros más concretos posibilita su tratamiento, pero resulta nefasta a la hora

\footnotetext{
${ }^{16}$ De alguna forma, la anterior configuración del sistema educativo basada en la Ley General de Educación del 70 incluía en sí misma una pequeña contradicción, por cuanto la escolarización se hacía obligatoria hasta los 14 años mientras que la "madurez social" suficiente para trabajar no se reconocía legalmente hasta los 16 , contradicción que expresa, en alguna medida, los problemas que la propia sociedad encuentra en definir quiénes son los niños, para qué, y en qué momento dejan de serlo.
} 
de tratar de "recomponer" los pedazos en orden a una mejor interpretación global del mismo. A ésta le ha seguido, no por ello menos importante, una fragmentación intradisciplinar, desde el mismo momento en que, como hemos visto, la infancia ha figurado como un epígrafe (y no siempre central) de los programas de docencia e investigación de la Sociología de la Educación, de la Familia o la propia Demografía. De esta forma, se aseguran las trabas a la hora de enfocar la infancia como construcción social para su estudio, de lo que resulta ese campo de estudio "difuso", vagamente nítido, del que vengo hablando hasta el momento. Veamos cuáles han sido las impresiones que, a este respecto, se recogen en uno de los pocos estudios que desde la sociología española han tratado el tema de la infancia de una forma rigurosa y global (Aguinaga y Comas, 1991: 3).

El análisis de los procesos sociales en los que se desarrolla, y transcurre, la evolución de la vida infantil hacia la condición de adulto apenas ha sido considerado desde la Sociología. No aparecen referencias bibliográficas, ni mucho menos una teoría establecida o un marco de referencia mínimo. Parecería, y éste es ya un dato de partida, que el espacio previo a la vida del adulto no es un espacio social, o al menos no ha sido analizado nunca como tal.

De alguna forma, ese espacio de lo no-social, pero que a su vez constituye el momento previo a la vida societal es el que ha sido entregado a otras visiones distintas de la puramente sociológica. El resultado es la ausencia de marcos teóricos y, por tanto, de definiciones. En su defecto, los propios agentes sociales proporcionan interpretaciones de la infancia que, a decir de los autores, siguen planteando importantes interrogantes (Aguinaga y Comas, 1991: 10)

Nuestros intentos de formalización han sido bastante inútiles porque el discurso social sobre el espacio de la ausencia en el que moran niños y adolescentes es a la vez un espacio muy bien delimitado y un espacio sin etapas formalmente diferenciadas. Lo que determina la evolución de niños y niñas, su ubicación en una determinada etapa, no son un conjunto de variables objetivas, sino el resultado de la definición que cada observador haga en su momento y ante cada ítem y que colocan al $<<$ observado ausente $>>$ en una posición determinada del eje dependencia autonomía.

Tal vez el propio problema radique en la naturaleza cambiante de la infancia, que merced a cambios demográficos y culturales ofrece rasgos muy distintos a través del tiempo.

En cualquier caso, no es menos cierto que existe una característica que constituye el núcleo del fenómeno, y que debería ser la base a través de la cual construyéramos un marco teórico sobre la infancia: la dependencia. De alguna forma, el hecho de que el niño es dependiente en alto grado de la estructura familiar (o, en su ausencia, de aquélla que hace las veces de familia) marca con tinte indeleble la condición de la niñez. Igualmente, se considera el momento en el que el grado de dependencia se atenúa como el comienzo de la adolescencia, que precede al momento en que la autonomía en términos sociales aparece en la vida de 
los individuos. Seguimos, por tanto, sin conocer una definición precisa que marque los límites cronológicos de la infancia, pero sabemos qué no es infancia, lo cual es un buen comienzo. Por otra parte, no podemos dejar pasar esta ocasión de señalar que la carencia de marco teórico y definiciones afecta incluso a herramientas básicas de la labor sociológica, como ocurre en el caso de la Enciclopedia Internacional de Ciencias Sociales (Reinhold y Bovard) ${ }^{17}$ que en la voz "infancia" recoge toda una serie de consideraciones sobre la maduración y desarrollo de los bebes, los efectos de la privación materna, etc. Sin aportar en ningún momento una base sólida que permita construir una definición adecuada del fenómeno, pese a que señala, en repetidas ocasiones, la invalidez de un modelo reduccionista biológico o psicológico a la hora de interpretar la naturaleza del niño como ser social desde su nacimiento. Igualmente, el carácter social de la infancia se ha revestido de una suerte de "invisibilidad" en los diccionarios de Ciencias de la Educación y Pedagogía, de tal forma que es posible detectar indicios que nos induzcan a pensar que la infancia posee, ante todo, una base social, pero éstos nunca cuajan en un enunciado formal sobre tal carácter social del fenómeno.

En definitiva, la infancia constituye un fenómeno societario, no exclusivamente previo a lo social, sino inserto en la propia estructura de las sociedades y construido en marcos de interacción social intersubjetiva, caracterizado:

a) por su dependencia ${ }^{18}$, en tanto que ésta viene a ser no sólo social sino física (o de subsistencia) durante los primeros años de vida y se centra, especialmente, en algún tipo de grupo primario o micro-comunidad, habitualmente una familia.

b) por su duración (dimensión temporal), en tanto en cuanto ésta es variable pero consistente, dado que supone un tiempo acotado en el que se produce la transición desde un nivel máximo de dependencia hasta el momento en que prima la autonomía del individuo, y que en la sociedad occidental podría coincidir con el propio período de escolarización obligatoria, aunque probablemente es más preciso concretarlo en torno a los 0-14 años.

c) por la identificación y uso que los propios agentes sociales hacen de ella, en la medida que la sociedad marca una diferencia entre niños y adolescentes

\footnotetext{
${ }^{17}$ Véase: Reinhold, H.L y Bovard, H.E., "Infancia",Enciclopedia Internacional de Ciencias Sociales, Madrid, Aguilar, pp. 769-780.

${ }^{18}$ Este aspecto de la dependencia implica, precisamente, una paradoja, que expresa con su ingenio habitual Gil Calvo al afirmar que "la lucha por el poder infantil o por la liberación de la infancia es algo impensable, pues el sometimiento de los menores a la tutela de los adultos es antropológicamente inevitable [... Y en este sentido, el único ejercicio legítimo de la autoridad adulta es el que busca como primer objetivo la emancipación de la infancia". (Gil Calvo, 1991: 139).
} 
que viene a tener su propia traducción en términos jurídicos, lingüísticos, culturales, etc... Esta última característica es fundamental, en tanto marca la medida de la diversidad de la infancia en distintos contextos culturales, y es requisito indispensable tenerla en consideración para cualquier tarea investigadora.

Una vez realizadas estas consideraciones será necesario aplicarlas en estudios e investigaciones que arrojen alguna luz sobre aquellos aspectos de la infancia que han permanecido ignorados, si bien es cierto que los niños han atraído la atención de los sociólogos y otros científicos sociales en el caso de la marginación y el comportamiento desviado. Ha sido en este campo en el que se ha producido una mayor sistematización de conocimientos en torno a la infancia, y tal vez la explicación a esta excepción ya la proporcionase Foucault en su estudio sobre el nacimiento de una tecnología del poder; afirma el pensador francés que a medida que el poder se vuelve más anónimo y funcional, aquellos sobre los que se ejerce tienden a estar más individualizados, de forma que "en un sistema de disciplina el niño está más individualizado que el adulto, el enfermo más que el hombre sano, el loco y el delincuente más que el normal y el no delincuente" (Foucault, 1975). El interés por los casos de marginación en la infancia sería alimentado, de nuevo, por esa visión pre-social de la situación del niño, en la medida que el corregir las situaciones de marginación supone aplicar una determinada tecnología social del poder en la prevención del futuro comportamiento desviado en el adulto. La biografía del niño, desde este punto de vista, es el paso necesario para elaborar una biografía de la sociedad, en la medida que todos los adultos albergan ese estado pre-social dentro de ellos.

Por último, una nueva cuestión ¿Debemos negar la posibilidad de un tratamiento parcial de la infancia desde otras disciplinas? La respuesta es rotundamente negativa. En primer lugar, hablar de infancia no es hablar de niños, puesto que esta última palabra remite a consideraciones más centradas en aspectos biológicos, psicológicos y médicos, aunque admite también un enfoque social. Ahora bien, hablar de la niñez o de la infancia ignorando lo que hay en ella de social constituye el error a evitar, y en la medida que podamos integrar diferentes disciplinas será más difícil cometerlo. Precisamente, el objetivo del siguiente y último apartado dentro de esta reflexión es el de construir una matriz de integración disciplinar que haga factible, en la medida de lo posible, dicha colaboración.

\section{Finalmente, un intento de integración disciplinar}

Si en algún momento pudiera haber dado la impresión de que el objeto de estas páginas ha sido el de "arrebatar" a la infancia de las miradas de otras disciplinas o ciencias, sociales o no, nos gustaría demostrar que se ha pretendido todo lo contrario. La existencia de otras visiones y otras voces sobre el mundo de la infancia no es sinónimo de desorden o caos metodológico, sino una última y genuina expresión de la propia complejidad y multidimensionalidad del fenóme- 
no a estudiar (y en última instancia, de la necesidad de mantener un pluralismo cognitivo y metodológico). En este sentido, no es necesario repartir, sino integrar, como creo haber afirmado ya en algún momento de este texto.

La presunción de la existencia de múltiples niveles de estudio de un mismo fenómeno implica distintas construcciones metodológicas del objeto, pero también hacernos una pregunta: ¿Cuál es el lugar de la Sociología en todo esto? Inevitablemente, la respuesta debe tender a la globalidad. La Sociología tiene la difícil, y hasta cierto punto ingrata misión de integrar aportaciones procedentes de otros campos de actividad científica en una concepción global de la infancia como fenómeno inserto en una estructura y dinámica social particular, de tal manera que se asegure que el objeto de estudio va a ser tratado en su totalidad y no parcialmente, como ha venido ocurriendo hasta el momento

Podría argumentarse, no sin lógica, que otras muchas disciplinas se ocupan de aspectos relacionados en mayor o menor medida con la infancia; sería el caso, como hemos visto al comenzar este ensayo, de la historia, pero también del trabajo social o la psiquiatría. No obstante, he preferido construir el gráfico incluyendo exclusivamente aquellas disciplinas o ciencias que pueden entrar en conflicto más fácilmente, atendiendo al conjunto de problemas y tendencias señaladas en párrafos anteriores.

Una forma mucho más detallada de construir principios de relación interdisciplinar podría estar basada en la construcción de matrices de integración, en las que figuren de forma desglosada aquellas parcelas de la realidad social que son susceptibles de estudios pluriparadigmáticos y en qué medida debería participar cada disciplina. Lógicamente, la construcción de este tipo de herramientas metodológicas parte siempre desde una determinada óptica, en este caso la de la problemática específica de la sociología. Por ello, lo que sigue no debe ser interpretado como un intento de encasillamiento definitivo, ni un reparto arbitrario de campos de estudio. Simplemente, he intentado facilitar una posible investigación multidisciplinar, al tiempo que el desarrollo en epígrafes en sentido descendente (desglosando éstos a partir de un campo genérico de estudio) allana el camino de cara a la elaboración de proyectos de investigación y programas enfocados a la actividad docente. En la siguiente matriz se recoge de forma abreviada: 1) una clasificación general de dimensiones a partir del problema genérico de la infancia (primera columna); 2) un desglose en epígrafes concretos de esas mismas dimensiones (segunda columna) que me parecen especialmente relevantes; 3) y finalmente, una relación de posibles disciplinas implicadas en el estudio de dicha dimensión, de forma que la integración disciplinar se convierte en un fenómeno cońcreto y operacionalizable, más allá de los marcos generales y las propuestas globales (tercera columna). 
Tabla 2.

Parcelación/Integración multidisciplinar en el estudio sociológico de la infancia.

\begin{tabular}{|c|c|c|}
\hline DIMENSIÓN DE ESTUDIO & OPERACIONALIZACIÓN EN EPÍGRAFES & $\begin{array}{l}\text { OTENCIALIDAD DE } \\
\text { RACIÓN DISCIPLINAR }\end{array}$ \\
\hline $\begin{array}{l}\text { LA INFANCIA COMO } \\
\text { CONSTRUCCIÓN SOCIAL }\end{array}$ & $\begin{array}{l}\text { El concepto de infancia: recorrido sociohistórico. Lo determinado y } \\
\text { lo indeterminado en el desarrollo infantil. La infancia como fenómeno } \\
\text { social. El estudio de la infancia desde una perspectiva sociológica. } \\
\text { La infancia y las distintas culturas. }\end{array}$ & $\begin{array}{l}\text { Historia, Historia Social, } \\
\text { Filosofia. }\end{array}$ \\
\hline $\begin{array}{l}\text { ASPECTOS METODOLÓGICOS } \\
\text { EN LA INVESTIGACIÓN SOCIAL } \\
\text { DE LA INFANCIA }\end{array}$ & $\begin{array}{l}\text { Fuentes secundarias para el estudio de la infancia. ¿Cuantitativo/Cualitativo? } \\
\text { Técnicas de investigación social aplicadas al estudio de la infancia. } \\
\text { Definición de indicadores sociales en el ámbito de los menores. } \\
\text { Censos, registros y otras fuentes. }\end{array}$ & $\begin{array}{l}\text { Estadística, Demografía, } \\
\text { Economía. }\end{array}$ \\
\hline $\begin{array}{l}\text { INFANCIA, DESARROLLO, } \\
\text { REPRODUCCIÓN SOCIAL Y } \\
\text { SOCIALIZACIÓN }\end{array}$ & $\begin{array}{l}\text { La "Naturaleza del niño" vs. Aprendizaje Social. La socialización como concepto } \\
\text { sociológico. Socialización, cultura y personalidad. La reproducción social, entre } \\
\text { lo individual y lo social. Infancia, crecimiento y reduccionismos. El desarrollo } \\
\text { desde una perspectiva sociológica. ¿Cómo aprendemos a ser seres sociales? }\end{array}$ & $\begin{array}{l}\text { Psicología social, } \\
\text { Psicología, } \\
\text { Antropología Social. }\end{array}$ \\
\hline $\begin{array}{l}\text { ASPECTOS DEMOGRÁFICOS } \\
\text { RELACIONADOS CON } \\
\text { LA INFANCIA }\end{array}$ & $\begin{array}{l}\text { La infancia y el cambio demográfico. Fecundidad, Fertilidad, Natalidad y pautas } \\
\text { demográficas de la Sociedad moderna. La medición de la reproducción social: } \\
\text { modelos demográficos. }\end{array}$ & $\begin{array}{l}\text { Demografía, } \\
\text { Estadística. }\end{array}$ \\
\hline FAMILIA E INFANCIA & $\begin{array}{l}\text { La familia y el cambio social: nuevas formas familiares y sus consecuencias } \\
\text { para la infancia. El hijo en la familia. Redefinición de las funciones familiares: } \\
\text { ¿hacia un nuevo modelo familiar? La infancia y las alteraciones del ciclo de } \\
\text { vida de la familia. Familia y socialización: hacia un modelo explicativo del } \\
\text { por qué y el cómo de la transmisión de valores y otros contenidos culturales. }\end{array}$ & $\begin{array}{l}\text { Psicología Social, } \\
\text { Antropología Social, } \\
\text { Psiquiatría, } \\
\text { Trabajo Social. }\end{array}$ \\
\hline
\end{tabular}


Tabla 2. (continuación)

Parcelación/Integración multidisciplinar en el estudio sociológico de la infancia.

\begin{tabular}{|c|c|c|}
\hline DIMENSIÓN DE ESTUDIO & OPERACIONALIZACIÓN EN EPÍGRAFES & $\begin{array}{l}\text { POTENCIALIDAD DE } \\
\text { RACIÓN DISCIPLINAR }\end{array}$ \\
\hline $\begin{array}{l}\text { LA INFANCIA EN EL MEDIO } \\
\text { EDUCATIVO }\end{array}$ & $\begin{array}{l}\text { La infancia y su socialización en la escuela. Modelos de atención a los } \\
\text { menores en el ámbito educativo. Educación y reproducción social. } \\
\text { La interacción en el aula: curriculum escolar y educación. Estructura social, } \\
\text { infancia y rendimiento escolar. El comportamiento desviado en la escuela, } \\
\text { ¿fracaso escolar o social? El rol del maestro ante los menores }\end{array}$ & $\begin{array}{l}\text { Pedagogía, Psicología } \\
\text { de la Educación, } \\
\text { Psicología Social. }\end{array}$ \\
\hline INFANCIA Y EXCLUSIÓN & $\begin{array}{l}\text { Infancia, pobreza y marginación. Conducta desviada e infancia. Maltrato infa } \\
\text { factores de rieso.Trastornos de la personalidad durante el desarrollo: sus cone } \\
\text { con la estructura social. Infancia y discapacidad. El menor y la delincuencia } \\
\text { infantil. El trabajo infantil. Prevención de desviaciones: redes de apoyo local }\end{array}$ & $\begin{array}{l}\text { Psicología Social, } \\
\text { es Trabajo Social, } \\
\text { Psiquiatría. } \\
\text { enor. }\end{array}$ \\
\hline $\begin{array}{l}\text { LA PROTECCIÓN SOCIAL } \\
\text { AL MENOR }\end{array}$ & $\begin{array}{l}\text { Política familiar y protección a la infancia. Modelos de intervención estatal. } \\
\text { El sistema público de servicios sociales en la atención al menor. El papel del } \\
\text { Estado en el desarrollo infantil vs. los derechos de la familia. }\end{array}$ & $\begin{array}{l}\text { Trabajo Social, } \\
\text { Politología, Economía. }\end{array}$ \\
\hline $\begin{array}{l}\text { INFANCIA Y } \\
\text { PRODUCCIÓN CULTURAL }\end{array}$ & $\begin{array}{l}\text { Infancia y comunicación. Los mass-media y la socialización de la infancia. } \\
\text { La literatura infantil: una aproximación sociológica. Modelos educativos } \\
\text { extraescolares: industria cultural y representación de la realidad social. } \\
\text { La sociedad del ocio y la infancia. El consumo y su relación con los menores }\end{array}$ & $\begin{array}{l}\text { Psicología Social, } \\
\text { Antropología Social, } \\
\text { Historia, } \\
\text { C.C. Comunicación }\end{array}$ \\
\hline
\end{tabular}




\section{CONCLUSIÓN}

¿Era posible organizar otro esquema distinto? Probablemente sí. Sin embargo, en aras de la coherencia, se ha tratado de que el producto final corresponda, al menos en la medida de lo posible, con las disquisiciones que hemos venido introduciendo desde las primeras líneas en éste que pretende ser, ni más ni menos, un instrumento de trabajo para todos aquellos que operen en el ámbito de la infancia. Dicho instrumento, como cualquier otro, siempre es mejorable, puesto que en la matriz anterior habrán quedado cosas en el tintero que otros considerarían centrales, de la misma forma que se habrán incluido algunas otras que pueden y deben ser objeto de discusión. Por supuesto, podría haber incluido entre las disciplinas que presentan un potencial grande de integración multidisciplinar -por poner un caso- a la biología, con relación a la noción de desarrollo del niño, buscando integrar el punto de vista tripartito físico-psicológico-social, al estilo de lo propuesto, con buen juicio, por Piaget (1977); sin embargo, se ha optado finalmente por no hacerlo para no complicar en exceso aquello que es sólo el comienzo de una tentativa en la que hay que andar con pie seguro, pero también prudente. En cualquier caso, el objetivo ha sido tan sólo ilustrar cómo:

a) la infancia es una construcción social, tal y como la entendemos hoy en nuestra sociedad. Es posible incluso, seguir la pista con las herramientas del historiador a ese proceso que configura el moderno concepto de infancia como un ámbito de sobreprotección, identificado con la idea de lo inocente, aquello que debe quedar a salvo del influjo externo o expuesto a éste de forma cuidadosamente dosificada.

b) Parcelando y dispersando un objeto de estudio no se consigue saber más, sino menos, y es por esto que he tratado de elaborar una guía general (quizás incluso muy vaga) del camino a través del cuál es posible saber más y mejor sobre la infancia.

c) Finalmente, he considerado la infancia, no cómo un estado pre-social, sino como un grupo de edad más, inserto de lleno en la propia estructura social, pero que posee la peculiaridad de constituir, a los ojos de la sociedad, un terreno moldeable en la medida que encarna la potencialidad del ser humano como ser social, indeterminado, aunque condicionado.

\section{BIBLIOGRAFÍA}

AGUINAGA, J. y D. COMAS (1991), Infancia y adolescencia: la mirada de los adultos, Madrid, Ministerio de Asuntos Sociales.

ANDERSON, M. (1982), Aproximaciones a la historia de la fanilia occidental, Madrid, Siglo $\mathrm{XXI}$. 
APPLE, M. (1996), Política cultural y educación, Madrid, Morata.

ARANGO, J. (1993), "El Sur en el sistema migratorio europeo. Evolución reciente y perspectivas", Política y Sociedad, n¹2, pp. 7-19.

ARIÈS, P. (1987), El niño y la vida familiar en el antiguo régimen, Madrid, Taurus.

BEJAR, H. (1990), El ámbito íntimo. Privacidad, individualismo y modernidad, Madrid, Alianza.

BELLAH, R.N. et al. (1989), Hábitos del corazón, Madrid, Alianza.

BELTRÁN, M. (1991), La realidad social, Madrid, Tecnos.

BURGUIÉRE, A. (1998), "Las mil y una familias en Europa”. En: Burguiére, A et. al. Historia de la familia, Madrid, Alianza.

C.E.C.S. (1994), España 1993. Una interpretación de su realidad social, Capítulo VIII "La protección social a la infancia", pp. 368-391, Madrid.

GOFFMAN, E. (1972), Internados. Ensayos sobre la situación social de los enfermos mentales, Buenos Aires, Amorrortu.

CORSARO, W. (1997), Sociology of Childhood, California, Pine Forge Press.

DAVILA, P. et al. (1991), "La protección infantil y los tribunales tutelares de menores en el Pais Vasco", Historia de la Educación, N 10, pp. 227-252.

DE MAUSE, Ll. (1982), Historia de la infancia, Madrid, Alianza.

DURKHEIM, E. (1990), Educación y Sociología, Barcelona, Península.

ELIAS, N. (1989), El proceso de la civilización, Mexico, FCE.

ERIKSON, E. (1993), Infancia y Sociedad, Buenos Aires, Lumen-Hormé.

FOCAULT, M. (1975), Vigilar y castigar, Madrid, Siglo XXI.

FREUD, S. (1970), El malestar en la cultura, Madrid, Alianza.

GIDDENS, A. (1995), La constitución de la sociedad. Bases para la teoría de la estructuración, Buenos Aires, Amorrortu.

GIL CALVO, E. (1991), "La emancipación de la infancia", I Congreso internacional Infancia y Sociedad, Madrid, Ministerio de Asuntos Sociales.

HUBER, J. and G. SPITZE (1988), "Trends in Family Sociology", en: Smelser, N.J. Handbook of Sociology, USA, Sage Publications. 
HUFTON, O. (1992), "Mujeres, Trabajo y familia" en: Duby, G. y Perrot, M. Historia de la mujeres, Madrid, Taurus.

IGLESIAS DE USSEL, J. (1985), «Socialización y Control Social», en Del Campo, S. (ed.). Tratado de sociología, Madrid, Taurus.

JACKSON, P. (1991), La vida en las aulas, Madrid, Morata.

JENKS, C. (1982), The Sociology of Childhood. Essential Readings, London, Gregg Revivals.

LEBRUN, F. (1998), "El cura, el príncipe y la familia”, en: Burguiére, A et. al. Historia de la familia, Madrid, Alianza.

LERENA, C. (1985), "Educación” en: Del Campo, S. (ed.). Tratado de sociología, Madrid, Taurus. (1986), Escuela, ideología y clases sociales en España, Barcelona, Ariel.

LEVI, G. y J. SCHMITT (1996), Historia de los jóvenes, Madrid, Taurus.

MORENTE MEJÍAS, F. (1996), "La familia ante la vulnerabilidad de la infancia”, Revista Internacional de Sociología (tercera época), N¹5, pp. 123-141.

(1997), Los menores vulnerables, Jaén, Universidad de Jaén.

ORTEGA, F. et. al. (1989), Manual de Sociologia de la Educación, Madrid, Visor.

PIAGET, J. (1977), Escritos Sociológicos, Barcelona, Ariel.

REINHOLD, H.L y H.E. Bovard, "Infancia", Enciclopedia Internacional de Ciencias Sociales, Madrid, Aguilar, pp. 769-780.

SENNETT, R. (1978), El declive del hombre público, Barcelona, Península.

SEGALEN, M. (1992), Antropología histórica de la familia, Madrid, Taurus.

VARELA, J. (1986), "Aproximación genealógica a la moderna percepción social de los niños", Revista de Educación, n² 281, pp. 155-175.

(1989), "Elementos para una genealogía de la escuela primaria en España", en: Ortega, Félix. et. al. Manual de Sociología de la Educación, Madrid, Visor.

VIÑAO, A. (1998), Tiempos escolares, tiempos sociales. La distribución del tiempo y del trabajo en la enseñanza primaria en España (1838-1936), Ariel, Barcelona. 
ABSTRACT. The study of childhood has not been a main score in sociological theory, althought its rol in the production and re-production of society is the base of all our social life in communities. This text tries to summarize some of the contributions to this topic in sociology, in order to remark the need for better sociological approaches to Childhood as a complex sociological object. It is also important to notice, as well as to understand, that the so called «childhood» is a social construction, becoming a privileged source of sociological analysis.

E-mail: ivan@uhu.es 